\title{
The physical properties of normal A stars
}

\author{
Saul J. Adelman \\ Department of Physics, The Citadel, 171 Moultrie Street, Charleston, SC 29409, USA email: \\ adelmans@citadel.edu
}

\begin{abstract}
Designating a star as of A-type is a result of spectral classification. After separating the peculiar stars from those deemed to be normal using the results of a century of stellar astrophysical wisdom, I define the physical properties of the "normal" stars. The hotter A stars have atmospheres almost in radiative equilibrium. In the A stars convective motions can be found which increase in strength as the temperature decreases.
\end{abstract}

Keywords. Stars: abundances, stars: fundamental parameters, stars: rotation, convection, diffusion, magnetic fields

\section{What are normal A stars?}

Each star examined in sufficient detail is unique. But astronomical progress requires that we try to group stars so that patterns emerge as functions of fundamental parameters. A century ago the Harvard College Observatory spectral classifiers defined A-type stars using order $100 \AA \mathrm{mm}^{-1}$ spectra. They have strong Balmer lines and many faint to moderately strong lines. The number of lines and degree of line blending in the photographic region grows rapidly towards the F stars. A stars lack the He I lines seen at classification dispersions in B stars. F stars have even more numerous and stronger metal lines. MK classifications use pairs of spectral lines in the photographic region. Morgan and some other spectral classifiers opened the slits of their spectrographs to minimize the differences due to rotation. So their resolutions are less than one would infer from their inverse dispersions. Stellar rotation makes weak lines disappear, causes blending, and affects the continuum location. Photometric criteria are used to define some A-star classes, but these violate the spirit of the pure classification process.

Normal single A stars can be completely classified or are normal white dwarfs. Understanding the marginal and peculiar stars may be an exercise in the physical processes involved with convection, diffusion, rotation, and magnetic fields. Many assert that the varieties of A stars are distinct from one another. However, many types probably merge into one another. Processes associated with some kinds of photometric variability just disturb the outer layers while leaving the other properties essentially alone. We exclude SB2 and those binary stars that have participated in a substantial mass exchange.

Charles Cowley defined the superficially normal stars as those whose classification spectra look normal, but are not with those used by abundance analyzers. Many MercuryManganese and marginal metallic-lined stars belong to this category. Finding such stars also depends on the signal-to-noise ratio of the spectra and on the effective temperature as the mean metallic line strength in the optical region increases with decreasing temperature in the A stars. If one can photometrically calibrate classification dispersion spectra as the ASTRA spectrophotometer (Adelman et al. 2004) will do, then one may be able to more easily separate these stars from the normal stars.

Are the classification standards well chosen? Abt \& Morrell (1995) note that some standards, in particular Vega and $\alpha$ Dra, are abnormal. Using them may result in similar 
stars being classified as normal. Further many normal stars near A2 IV have characteristics similar to those of peculiar stars and the mean rotational velocities of normal stars are depressed for those types where Am and Ap stars are most frequent. Sharp-lined and broad-lined standards for a given spectral type may not be equivalent.

The hotter main sequence band A stars have almost purely radiative atmospheres. In the mid-A stars convection begins to play a role in energy transport. The degree to which this happens increases with decreasing temperature and decreasing surface gravity.

Old metal-poor evolved A-type Population II stars often are horizontal-branch stars, e.g., RR Lyrae and the Field Horizontal Branch A Stars, which are found in globular clusters and the Galactic halo. Some Population I A stars are metal poor relative to the Sun, e.g., Vega and $\alpha$ Dra (Adelman \& Gulliver 1990; Adelman et al. 2001), but many more should be known. Abt \& Morrell (1995) suggest as candidates superficially normal stars with low rotational velocities and weak Mg II $\lambda 4481$ and Ca II K lines. The most extreme metal-poor stars, the $\lambda$ Boo stars (Morgan et al. 1943, Hauck \& Slettebak 1983, Gray 1988), are extremely obvious at classification dispersions. Attempts to define them as a tightly defined group of rapidly rotating metal-poor stars (see, e.g., Gerbaldi et al. 2003) have problems. It might be more useful to look at the all metal poor A stars and recognize ways they are related to the prototype. Fast rotators seen nearly pole-on are not necessarily metal-poor (Gulliver, Hill \& Adelman 1994), e.g, $\nu$ Cap.

Some main sequence band chauvinists exclude from the normal stars those outside of the main sequence band and not deriving all of their radiated energy from the conversion of hydrogen into helium in their cores. Pre-main sequence stars are evolving towards the Zero Age Main Sequence. Population I A supergiants evolved from more massive progenitors than main sequence band stars. Dredge-up events may have affected their envelope abundances especially of $\mathrm{C}, \mathrm{N}$, and $\mathrm{O}$. Photospheric abundances can be altered by mass exchange events during the evolution of binary stars.

Some A stars have nearby circumstellar or interstellar material. For example, $\beta$ Pic and Vega have outlying dust disks surrounding them, perhaps as remnants of star formation. Hot inner disks around rapidly rotating B and A dwarf or shell stars are due to material accreted from the interstellar medium in dense interstellar regions (Abt 2004). Also the Be star sequence extends into the early A stars, the Herbig Ae stars.

The magnetic CP (mCP) (Preston's (1974) CP2) stars include those peculiar B and A stars with detected magnetic fields. Many show spectrum, photometric, and magnetic variability. Stars with similar spectra showing abnormally strong lines of $\mathrm{Si}, \mathrm{Cr}, \mathrm{Sr}$, and the rare earths are considered class members especially if they exhibit variability.

Roman et al. (1948) defined the Am or metallic-lined (Preston's (1974) CP1) stars as having Sp type (Ca II K line) < Sp type (Balmer lines) < Sp type (metallic lines). Conti (1970) extended the class to include stars with weak Sc II lines in place of or in addition to a weak K line. The Mercury-Manganese (HgMn) (Preston's (1974) CP3) stars are late peculiar B stars for which Hg II $\lambda 3984$ and/or Mn II lines are the most prominent enhanced features. These types form a sequence of non-magnetic CP stars, but some have speculated that they have far more complex fields than the usual mCP stars. Identifying those with some rotation can be difficult. Recently Adelman et al. (2003) showed that the coolest HgMn stars evolve into the hottest Am stars. This unifies these classes and provides a test for theoreticians concerning the abundance of mercury. There is a full range of abundance patterns from the marginal Am stars whose abundances are merely suggestive of peculiarity through those who show the classical Am abundance pattern. Similar effects should occur for the HgMn stars. But due to the paucity of lines in optical region spectra, this may well be a project for high dispersion ultraviolet spectroscopy. 
Table 1. Parameters of normal main sequence stars

\begin{tabular}{ccccccccc}
\hline Sp. Type & $B-V$ & $b-y$ & $\begin{array}{c}T_{\text {eff }} \\
(\mathrm{K})\end{array}$ & $M_{\mathrm{V}}$ & $\begin{array}{c}\text { Mass } \\
\left(M_{\odot}\right)\end{array}$ & $\begin{array}{c}\text { Radius } \\
\left(R_{\odot}\right)\end{array}$ & $\begin{array}{l}\log g<v \sin i> \\
\left(\mathrm{km} \mathrm{s}^{-1}\right)\end{array}$ \\
\hline A0 & 0.000 & 0.00 & 9727 & 0.7 & 2.40 & 1.87 & 4.27 & 149 \\
A2 & 0.055 & 0.04 & 8820 & 1.3 & 2.19 & 1.78 & 4.28 & 145 \\
A5 & 0.143 & 0.09 & 7880 & 2.0 & 1.86 & 1.69 & 4.25 & 137 \\
A6 & 0.170 & 0.10 & 7672 & 2.1 & 1.80 & 1.66 & 4.25 & 134 \\
A7 & 0.198 & 0.12 & 7483 & 2.3 & 1.74 & 1.63 & 4.25 & 132 \\
A8 & 0.228 & 0.14 & 7305 & 2.4 & 1.66 & 1.60 & 4.25 & 128 \\
A9 & 0.265 & 0.16 & 7112 & 2.5 & 1.62 & 1.55 & 4.26 & 124 \\
F0 & 0.300 & 0.17 & 6949 & 2.6 & 1.55 & 1.51 & 4.27 & 117 \\
\hline
\end{tabular}

Abt \& Morrell (1995) noted that virtually all Am and mCP stars have equatorial rotational velocities $<120 \mathrm{~km} \mathrm{~s}^{-1}$ while normal A0-F0 main sequence stars have larger values. The latter include those with weak $\mathrm{Ca}$ II $\mathrm{K}$ lines or belong to short-period binaries. Abt (2000) concluded that rotation alone can explain the appearance of an A star at classification dispersions as either normal or abnormal. The few sharp-lined normal A stars are just fast rotators seen nearly pole-on. Gulliver et al. (1994) gave a few examples that exhibited weak line profiles with flat cores.

Preston's (1974) CP4 or Helium-weak stars are peculiar B stars whose He I line strengths are weaker than one would expect based on their $U B V$ colors. They are a mixture of hotter analogues to the mCP (CP2) and to the HgMn (CP3) stars.

The classical variability strip crosses the main sequence between A2 V and F0 V (Wolff 1983). There is a paucity of sharp-lined normal stars here. Observations from the Earth show that $1 / 3$ of these main sequence band stars are $\delta$ Scutis. Recent space observations suggest (Breger 2004) that the rest are variable with smaller amplitudes. Besides the $\delta$ Scuti stars, the roAp stars and the $\gamma$ Dor stars also pulsate with each class having its own driving mechanism. The peculiar roAp stars have abnormal abundance distributions. They are cooler than most of the other mCP stars (Ryabchikova, private communication). Members of the two classes with solar type abundances should be considered normal stars. Waelkens et al. (1998) proposed an analogous strip to connect the $\beta$ Cep stars, the slowly pulsating B stars, and the $\alpha$ Cyg variables.

Thus normal A stars have surficial abundances close to "solar" (supergiants and white dwarfs excepted), lack detectable magnetic fields and emission lines, have equatorial rotational velocities $>120 \mathrm{~km} \mathrm{~s}^{-1}$, and have not participated in a substantial mass exchange event with a companion.

\section{Properties of main sequence band normal A stars}

Table 1 extracted from Gray (1992) provides a framework of useful values for main sequence A stars. Some sources cited later use slightly different values. In part this reflects differences between how the selections are made. Averages of the type given in Table 1 are absent for luminosity class III and IV A stars. Only the Sun is sufficiently close that we can determine its fundamental properties with good accuracies and precisions. Recently substantial progress has been and is continuing to be made for other nearby stars including main sequence band A stars. The Hipparcos satellite improved our knowledge of parallaxes. Most recent improvements in parallaxes made from the ground relate to stars not observed by Hipparcos.

Hipparcos photometry is a consistent database with order 100 observations of a large number of relatively bright stars with classified spectra. Adelman (2001) investigated 
the mean amplitude as a function of MK spectral type of apparently single normal stars in the Bright Star Catalogue (Hoffleit \& Warren 1991). The most stable stars (ampl. $<0.026$ mag.) were spectral types B9-A8 III and F0 III, B9-F0 IV, and B9A3 V, A5 V, and A6 V. Then come A4 V, A8-F0 V, and A9 III stars, and the B9 II and A6-F0 II supergiants (ampl. 0.027-0.032 mag.), A0-A5 II and A5-F0 I supergiants (ampl. 0.035-0.036 mag.), and finally B9-A4 I supergiants (ampl.> 0.050 mag.). The increase in the mean amplitudes of late A dwarfs reflects that they are low amplitude $\delta$ Scuti stars. Other major photometric databases exist especially for faint stars. Smallautomated telescope observers usually perform differential photometry of variable stars. Problems with comparison stars are often obliquely noted in publication. I use those near the variable on the sky with similar colors and small Hipparcos photometric data amplitudes (0.01-0.02 mag.). At best their ground-based standard deviations of the mean values in Strömgren uvby photometry are $<0.005$ mag. However, some stars that were constant for the three years of Hipparcos observations may be variable now.

Photometrically constant normal A stars which are not in binary systems have constant radial velocities. Such stars also lack detectable magnetic fields (Shorlin et al. 2002). Substantial mass loss does not occur in the main sequence band.

Eclipsing binaries have directly measurable rotational velocities. But for most stars, the inclination angles are unknown. Only for a few single normal A stars with peculiar flatbottomed profiles of weak lines such as Vega (Gulliver, Hill \& Adelman 1994) can we find the equatorial velocity exactly. Modelling shows $v_{\mathrm{eq}}=160 \pm 10 \mathrm{~km} \mathrm{~s}^{-1}$ and $i=7.9 \pm 0.5^{\circ}$ (Hill, Gulliver \& Adelman 2004). Several other stars can be similarly analyzed.

I concur with Abt (2000) that an equatorial velocity less than some particular value guarantees that a star is peculiar. But some fast rotating A stars are peculiar, e.g. CU Vir and 56 Ari. He I $\lambda 4471$ and $\mathrm{Mg}$ II $\lambda 4481$ are not the best lines for measuring $v \sin i$ as they are blends. Gray's (1992) use of Fourier techniques is very elegant, but requires high signal-to-noise and resolution. I fit clean single metal lines on the linear part of the curve of growth with Gaussian or rotational profiles and correct for the instrumental profile (using, e.g., VLINE (Hill \& Fisher 1986)) or synthesize the spectrum, rotate, and convole it with the instrumental profile (with a spectrum synthesis program such as SYNTHE (Kurucz \& Avrett 1981). Still the photospheric and outer envelope rotation rates do not necessarily tell us how fast the core rotates as the degree of coupling is uncertain.

Whether a star rotate as a solid body or differentially relates to the onset of atmospheric convection. When Abt \& Morrell (1995) deconvolved their rotational velocities, normal A0-A1 V, A2-A4 V, and A5-F0 V stars had peak rotational velocity distributions at about 225,190 , and $170 \mathrm{~km} \mathrm{~s}^{-1}$, respectively. For other luminosity classes these distributions have smaller mean values since as the stars become more luminous, their radii increase and angular momentum is conserved. Between spectral types A4-F0 V there may not be any sharp-lined normal stars (see, e.g., Abt \& Moyd 1973). Abt (2003) studied B and A dwarfs and giants. Combining normal and peculiar types, he found a statistically constant $v \sin i$ at $127 \pm 15 \mathrm{~km} \mathrm{~s}^{-1}$ for B0-A5 V stars. Then using interiors models, he predicted the giants' rotational velocities and obtained good results when he assumed rigid-body rotation. Reiners \& Royer (2004) investigated spectral type A0-F1 stars with $v \sin i$ values between 60 and $160 \mathrm{~km} \mathrm{~s}^{-1}$. For 74 stars using a Fourier technique they found results consistent with rigid-body rotation. One star was probably seen near poleon. Three stars appear to be rotating differentially with the spectral type of the earliest of these being A6.

The middle to late A-type stars show nonexistent to moderately strong chromospheric activity (see, e.g. Simon \& Landsman's (1991) investigation of the C II $\lambda 1335$ emission line). Using He I $\lambda 5876$ as an activity indicator in the Hyades, Praesepe, and Coma 
Clusters, the earliest stars detected range from $B-V=0.26$ (Hyades) to $B-V=0.31$ (Praesepe). But not all stars close to these in temperature are active (Rachford 1998). Schmitt (1997) studied X-ray data and found coronal emission is universal for spectral types A7 to G9 suggesting an onset of activity occurs near spectral type A7. Turner-Bey et al. (2003) found using Chandra satellite data that main sequence stars become X-ray emitters at $V-R=0.15$, corresponding to spectral types A6 to A9. Simon \& Landsman (1997) using HST/Goddard High Resolution Spectrograph) found N v $\lambda 1239$ transition region emission for the $\mathrm{A} 7 \mathrm{~V}$ stars $\alpha$ Aql and $\alpha$ Cep. They and $\tau^{3}$ Eri (A4 IV) $\left(T_{\text {eff }}\right.$ $=8200 \mathrm{~K}$ ) have chromospheric emission in Si III $\lambda 1206$. The later is the hottest main sequence band star with a chromosphere and hence an outer convection zone.

Am stars have a high while the mCP stars a low binary frequency. Abt (1965) who studied 55 normal A4-F2 IV and V stars did not find any binary with a period of less than 100 days. Still the frequency of binaries with long periods may be normal. Abt \& Bidelman (1969) found some normal A-type stars in binaries with $\mathrm{P}<2.5$ days.

Monnier (2003) reviews optical stellar interferometers for measuring angular diameters $(\theta)$. Lunar occultations have also been used for this purpose. With a parallax one can derive the radius. Bright stars with large $\theta$ 's are currently studied. van Belle et al. (2001) measured the oblateness of Altair (A7 IV-V) $\left(v \sin i=210 \pm 13 \mathrm{~km} \mathrm{~s}^{-1}\right.$; axial ratio $=$ 1.140). Both Altair and Vega (Gulliver et al. 1994) indicate that rotating single stars are ellipsoids of revolution with smaller polar than equatorial radii. These effects may not be small for the fastest rotating normal stars, in which case one should give both polar and equatorial values. For Vega whose $T_{\text {eff }}$ range is $350 \mathrm{~K}$, if one uses mean values of $T_{\text {eff }}$ and $\log g$ for a model atmosphere to derive elemental abundances the results will usually be close to those derived using spectral synthesis, but cannot reproduce the weak line profiles.

Astronomers can derive stellar parameters with and without the use of model atmospheres. The later leads to fundamentally determined values. Fundamental values of $T_{\text {eff }}$ (the effective temperature) can be obtained from measurements of the angular diameter and of the total integrated flux $\left(f_{\text {earth }}\right)$ of a star. We use these to obtain the total emergent flux at the stellar surface $F_{\text {star }}=4 f_{\text {earth }} / \theta^{2}$ and thus $T_{\text {eff }}=\left(F_{\text {star }} / \sigma\right)^{1 / 4}$ where $\sigma$ is the Stefan-Boltzmann constant. Alternatively rearranging in terms of directly observable quantities $T_{\text {eff }}=2341\left(F_{\text {bol }} / \theta_{R}^{2}\right)^{1 / 4}$ where $F_{\text {bol }}$ is the total bolometric flux (in $10^{-8}$ ergs $\mathrm{cm}^{-2} \mathrm{~s}^{-1}$ ) and $\theta_{R}$ is the angular diameter in milli-arcseconds.

Code et al. (1976) combined angular diameter measurements with ultraviolet, optical, and infrared fluxes to get such temperatures (see Table 2). Sirius ( $\alpha$ CMa) might be anomalous. The 2MASS and DENIS surveys contain new consistent infrared fluxes. Bohlin \& Gilliand (2004) give values on the HST White Dwarf flux scale from the far-uv to the ir and show discrepancies relative to the fundamentally calibrated values in the optical region. This pragmatic approach dependent on a theory may well be correct. Although they have achieved repeatability, systematic errors may be more important than random errors. Pagano et al. (2004) provides solar flux errors for a variety of instruments that are much greater than the White Dwarf flux method results indicate. I prefer direct calibrations with errors derived from the measurements for all spectral regions. The $\log g$ values for Table 2 are from Smalley \& Dworetsky (1995). The value for $\epsilon$ Sgr is larger than expected and with respect to values in Table 1 three of five single luminosity class $\mathrm{V}$ stars have evolved beyond the ZAMS. To supplement these values I use binary star values from Smalley et al. (2002).

The optical region fluxes at selected wavelengths use the Hayes \& Latham (1975) or Tüg, White \& Lockwood (1977) calibrations of Vega which are at best good to 1\%. Other grating scanner measurement errors are due to limited signal-to-noise and the use of 
Table 2. Some representative effective temperatures and surface gravities

\begin{tabular}{llll}
\hline Name & Sp. Type & $T_{\text {eff }}(\mathrm{K})$ & $\log g$ \\
\hline$\alpha$ Car & F0II & $7520 \pm 460$ & 1.50 \\
$\epsilon$ Sgr & B9.5III & $9420 \pm 240$ & 4.50 \\
$\alpha$ Oph & A5III & $7960 \pm 330$ & 3.80 \\
$\gamma$ Gem & A0 IV & $9220 \pm 330$ & 3.50 \\
$\beta$ Car & A2IV & $9150 \pm 240$ & 3.50 \\
$\beta$ Lyr & A0Va & $9600 \pm 180$ & 4.00 \\
$\alpha$ CMa & A1Vm & $9940 \pm 210$ & 4.33 \\
$\beta$ Leo & A3 V & $8870 \pm 350$ & 4.00 \\
$\alpha$ PsA & A3 V & $8760 \pm 310$ & 4.20 \\
$\alpha$ Aql & A7 V & $7990 \pm 210$ & 4.00 \\
& & & \\
$\beta$ Aur A & A1V & $9131 \pm 257$ & 3.93 \\
$\beta$ Aur B & A1V & $9015 \pm 182$ & 3.96 \\
V624 Her A & A3m & $8288 \pm 497$ & 3.83 \\
V624 Her B & A7V & $8092 \pm 474$ & 4.02 \\
RS Cha A & A8V & $7525 \pm 307$ & 4.05 \\
RS Cha B & A8V & $7178 \pm 225$ & 3.96 \\
$\gamma$ Vir A & F0V & $7143 \pm 451$ & 4.21 \\
$\gamma$ Vir B & F0V & $7143 \pm 451$ & 4.21 \\
\hline
\end{tabular}

Table 3. Masses, radii, and bolometric magnitudes of normal main sequence stars

\begin{tabular}{cccc}
\hline Sp. Type & Mass $\left(M_{\odot}\right)$ & Radius $\left(R_{\odot}\right)$ & $M_{\text {bol }}$ \\
\hline B9.5 & 2.38 & 2.17 & 0.68 \\
A0 & 2.24 & 2.09 & 0.97 \\
A1 & 2.14 & 2.03 & 1.18 \\
A2 & 2.04 & 1.97 & 1.41 \\
A3 & 1.98 & 1.92 & 1.57 \\
A4 & 1.92 & 1.88 & 1.72 \\
A5 & 1.86 & 1.84 & 1.88 \\
A6 & 1.79 & 1.79 & 2.04 \\
A7 & 1.74 & 1.75 & 2.19 \\
A8 & 1.66 & 1.69 & 2.42 \\
A9 & 1.58 & 1.62 & 2.67 \\
F0 & 1.50 & 1.56 & 2.90 \\
\hline
\end{tabular}

mean extinction coefficients. John Lester's Fourier Transform Spectrophotometer should provide absolute calibrations without the systematic errors of previous work. I hope he will calibrate a grid of stars. The ASTRA spectrophotometer (Adelman et al. 2004) should perform fast accurate absolute spectrophotometry for $\lambda \lambda 3300-9000$ using a grid of well-calibrated standards, only one of which initially has to be absolutely calibrated.

Binary star studies can lead to masses and also for eclipsing systems radii. Popper (1980) and Andersen (1991) provide lists of eclipsing binary stars with both stars near the main sequence with well-determined masses and radii. Detached main-eclipsing systems whose spectral lines are at times well separated relative to their widths and with light curves having two well-defined minima, provide the greatest number of reliable masses and radii. There are a also a few visual binaries with well-determined masses and radii. Table 3 is from Harmanec's (1988) summary based on modern binary star data.

As the radii of components can be determined in binary systems, one can find the apparent stellar diameters if we know the distance. Fundamental values of $T_{\text {eff }}$ can be obtained by determining the relative contributions of both components to the observed 


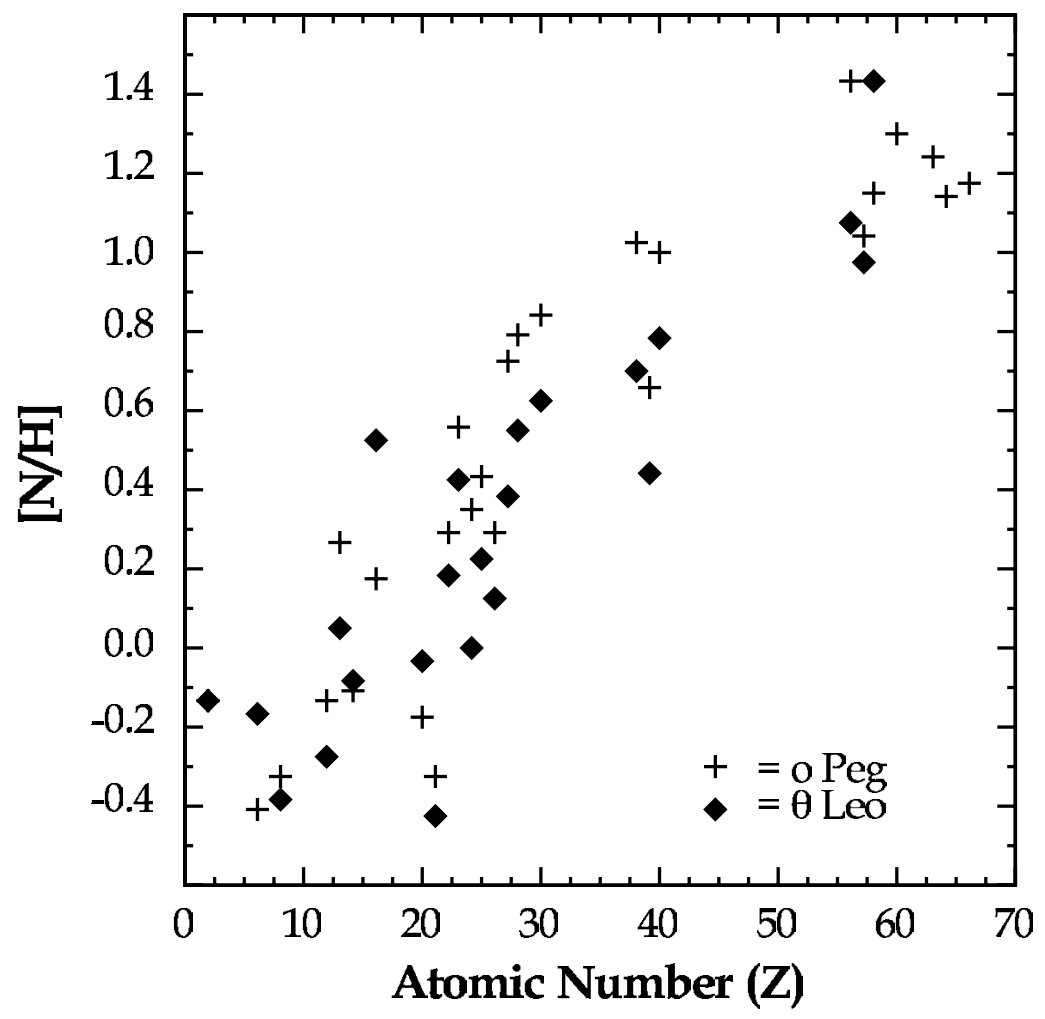

Figure 1. The abundance anomalies of the hot Am stars $o$ Peg and $\theta$ Leo relative to solar values $[\mathrm{N} / \mathrm{H}]$ as a function of atomic number $Z$.

flux. Smalley \& Dworetsky (1995) studied 4 systems while Smalley et al. (2002) an additional 11. With better spectrophotometric fluxes many more systems can be studied. If one knows the mass and the radius for a star then the surface gravity $g=G M / R^{2}$.

If both fundamental and non-fundamental values of the effective temperatures and surface gravities for stars agree or the offsets are well determined, then many workers will use non-fundamental techniques as they are easier to perform. One matches the observed flux distribution and Balmer line profiles with those predicted by model atmospheres. For stars earlier than about B9, the energy distribution gives the temperature and the Balmer line profiles the surface gravity. For spectral types A4 and cooler the roles are reversed. For B9-A3 stars some ambiguity arises, but via iteration using both fluxes and Balmer line profiles satisfactory fits can be found.

The IRFM (infrared flux method) (Blackwell \& Lynas-Gray 1994) can determine the effective temperature, $T_{\text {eff }}$, and angular diameter, $\theta$ of a star, based on absolute measurements of stellar monochromatic fluxes in the infrared region, $\mathrm{F}_{\lambda}$, and the integrated flux $\mathrm{F}$. If $\mathrm{R}$ is the ratio of the integrated to monochromatic flux, then when there is no interstellar absorption, one can find the stellar temperature and angular diameter from

$$
R=F / F_{\lambda}=\sigma T_{\text {eff }}^{4} / \Phi\left(T_{\text {eff }}, g, \lambda, \mathrm{A}\right) \text { and } F=\theta^{2} \sigma T_{\text {eff }}^{4} / 4
$$

where $\Phi\left(T_{\text {eff }}, \mathrm{g}, \lambda, \mathrm{A}\right)$ is the monochromatic emergent flux from a photosphere which is a function of the effective temperature $\left(T_{\text {eff }}\right)$, surface gravity $(g)$, wavelength of the monochromatic flux $(\lambda)$, and the atomic abundances (A) determined by model atmosphere theory. The IRFM is designed for maximum accuracy in finding $T_{\text {eff }}$ and $\theta$, as $\theta$ values with parallaxes can lead to absolute stellar radii for studies of stellar evolution. One 
Table 4. Stellar parameters

\begin{tabular}{|c|c|c|c|c|c|c|c|c|}
\hline Star & $\begin{array}{l}\text { Spectral } \\
\text { Type }\end{array}$ & $T_{\text {eff }}$ & $\log g$ & mean $Z$ & $\log \mathrm{Si} / \log \mathrm{Sr}$ & $\log \mathrm{Fe} / \mathrm{H}$ & $\begin{array}{r}v \sin i \\
{[\mathrm{~km} \mathrm{~s}}\end{array}$ & $\left.{ }^{-1}\right]^{\xi}$ \\
\hline 29 Cyg & $\mathrm{A} 7 \mathrm{Vp}$ & 7900 & 3.75 & $-1.50 \pm 0.46$ & 6.31 & -6.34 & 80 & 3.2 \\
\hline Vega & A0Va & 9400 & 4.03 & $-0.60 \pm 0.14$ & & -5.08 & 21 & 0.6 \\
\hline$\gamma$ Lyr & B9III & 9550 & 2.75 & $-0.39 \pm 0.40$ & 4.87 & -5.41 & 70 & 0.8 \\
\hline$\sigma$ Boo & $\mathrm{F} 2 \mathrm{~V}$ & 6744 & 3.88 & $-0.31 \pm 0.38$ & 4.85 & -5.86 & 8 & 1.6 \\
\hline$\alpha$ Dra & A0III & 10000 & 3.60 & $-0.23 \pm-0.26$ & 4.72 & -4.56 & 25 & 0.1 \\
\hline$\eta$ Lep & F1V & 6850 & 4.05 & $-0.22 \pm-0.36$ & 4.83 & -4.98 & 13 & 3.8 \\
\hline$\iota$ And & B9V & 11600 & 3.35 & $-0.19 \pm-0.15$ & & -4.68 & 60 & 1.3 \\
\hline 28 And & A7III & 7358 & 4.65 & $-0.16 \pm 0.24$ & 4.68 & -4.24 & 18 & 3.5 \\
\hline 14 Cyg & B9III & 10750 & 3.55 & $-0.12 \pm 0.23$ & 4.54 & -4.57 & 31 & 0.0 \\
\hline 134 Tau & B9IV & 10825 & 3.88 & $-0.09 \pm 0.16$ & 4.63 & -4.72 & 27 & 0.0 \\
\hline $29 \mathrm{Vul}$ & A0IV & 10200 & 4.10 & $-0.09 \pm 0.33$ & 4.49 & -3.8 & 49 & 1.2 \\
\hline$\epsilon \mathrm{Aqr}$ & $\mathrm{A} 1.5 \mathrm{~V}$ & 9050 & 3.75 & $-0.08 \pm 0.35$ & 4.60 & $-5.32:$ & 110 & 2.8 \\
\hline 2 Lyn & A1Va & 9295 & 4.10 & $-0.04 \pm 0.20$ & 4.65 & -4.51 & 44 & 2.1 \\
\hline$\alpha \operatorname{Sex}$ & B9.5III & 9875 & 3.55 & $-0.03 \pm 0.18$ & 4.50 & -4.46 & 21 & 0.3 \\
\hline$\nu$ Cap & B9.5V & 10250 & 3.90 & $-0.02 \pm 0.23$ & 4.53 & -4.52 & 23 & 0.0 \\
\hline 21 Lyn & A0mA1V & 9500 & 3.75 & $-0.02 \pm 0.37$ & $4 .($ & & 18 & 1.6 \\
\hline$\kappa$ Сер & B9III & 10325 & 3.70 & $-0.01 \pm 0.20$ & 4.56 & -4.23 & 23 & 0.3 \\
\hline HR 6559 & A7IV & 7900 & 3.20 & $0.07 \pm 0.40$ & 4.70 & -3.71 & 18 & 3.5 \\
\hline$\gamma$ Gem & A0IV & 9260 & 3.60 & $0.09 \pm 0.23$ & 4.36 & -4.78 & 10 & 2.0 \\
\hline HD 60825 & A0 & 8750 & 3.59 & $0.12 \pm 0.16$ & $\ldots$ & -4.41 & 14 & 2.5 \\
\hline 5 Aqr & B9.5III & 11150 & 3.35 & $0.13 \pm 0.20$ & $\ldots$ & -4.27 & 25 & 0.7 \\
\hline$\beta \mathrm{UMa}$ & A0mA1IV-V & 9600 & 3.83 & $0.14 \pm 0.40$ & 4.31 & & 45 & 2.5 \\
\hline 60 Leo & $\mathrm{Am}$ & 9250 & 4.25 & $0.15 \pm 0.35$ & 4.04 & -3.32 & 17 & 3.4 \\
\hline 7 Sex & A0Vs & 10135 & 3.69 & $0.17 \pm 0.38$ & 4.27 & -4.72 & 22 & 1.8 \\
\hline $15 \mathrm{Vul}$ & $\mathrm{Am}$ & 7700 & 3.50 & $0.19 \pm 0.47$ & 4.74 & -3.99 & 10 & 4.0 \\
\hline$\sigma$ Aqr & A0IV & 10125 & 4.00 & $0.24 \pm 0.43$ & 4.32 & -3.59 & 21 & 1.0 \\
\hline$\nu \mathrm{Cnc}$ & A0III & 10375 & 3.50 & $0.25 \pm 0.88$ & 4.51 & -3.42 & 13 & 0.1 \\
\hline$\theta$ Leo & $\mathrm{A} 2 \mathrm{~V}$ & 9325 & 3.65 & $0.25 \pm 0.51$ & 4.38 & -3.79 & 22 & 2.2 \\
\hline$\theta$ And & A2IV & 9000 & 4.00 & $0.26 \pm 0.40$ & 4.22 & -4.16 & 93 & 3.6 \\
\hline$\omega \mathrm{UMa}$ & A0IV-V & 10026 & 3.88 & $0.27 \pm 0.63$ & 4.27 & -3.40 & 46 & 1.1 \\
\hline $68 \mathrm{Tau}$ & A2IV-Vs & 9000 & 4.00 & $0.40 \pm 0.52$ & 4.16 & -3.43 & 9 & 2.3 \\
\hline$\epsilon$ Ser & $\mathrm{Am}$ & 8422 & 4.30 & $0.44 \pm 0.54$ & 4.26 & -3.93 & 33 & 5.2 \\
\hline 32 Aqr & $\mathrm{Am}$ & 7700 & 3.65 & $0.44 \pm 0.63$ & 4.42 & -3.72 & 6 & 4.5 \\
\hline$\pi$ Dra & A2A3IV & 9125 & 3.80 & $0.46 \pm 0.66$ & 4.18 & -3.42 & 26 & 3.5 \\
\hline$o$ Peg & A1IV & 9535 & 3.74 & $0.49 \pm 0.60$ & 4.21 & -3.47 & 6 & 1.3 \\
\hline$\phi$ Aql & A1IV & 9534 & 4.05 & $0.54 \pm 0.74$ & 4.09 & -3.99 & 28 & 3.1 \\
\hline $6 \mathrm{Lyr}$ & $\mathrm{Am}$ & 8155 & 3.90 & $0.60 \pm 0.70$ & 4.10 & -3.59 & 31 & 5.6 \\
\hline$\lambda \mathrm{UMa}$ & A1IV & 9000 & 3.75 & $0.66 \pm 0.66$ & 4.26 & -3.31 & 50 & 2.8 \\
\hline 59 Her & A3III & 9325 & 3.65 & $0.79 \pm 0.61$ & 4.08 & -3.62 & 27 & 2.8 \\
\hline
\end{tabular}

begins with the apparent visual magnitude and the effective temperature. The apparent visual magnitude is converted to the absolute visual magnitude using the distance (a Lutz-Kelker (1973) correction may need to be applied). The luminosity ( $\left.L=4 \pi R^{2} \sigma T_{\text {eff }}^{4}\right)$ is found by applying a Bolometric Correction (see, e.g., Bessell, Castelli \& Plex 1998) to account for the energy not included in the visual magnitude. Some widely used evolutionary tracks for solar composition stars $(Z=0.019, Y=0.273)$ are those of Girardi et al. (2000) and $(Z=0.020)$ Schaller et al. (1992).

Landstreet (1998) obtained the spectra of several ultra-sharp-lined stars at high resolution and signal-to-noise ratios. He found that the A1 V star HD 72660 with $\xi=2.2$ $\mathrm{km} \mathrm{s}^{-1}$ and two Am stars with much larger values $\left(4\right.$ to $5 \mathrm{~km} \mathrm{~s}^{-1}$ ) had strong spectral lines 
with significant asymmetries while hotter stars did not. Such effects must be accounted for in the analyses of stars where they are found.

Table 4 summarizes A star abundances and gives the stellar name, spectral type, effective temperature, surface gravity, mean metallicity with respect to the solar, the $\log \mathrm{Fe} / \mathrm{H}$ value, the $\log \mathrm{Si} / \log \mathrm{Sr}$ ratio, $v \sin i$ and the microturbulence $\xi$. Values are from Adelman (1986, 1987, 1989, 1991, 1994, 1996, 1999), Adelman \& Albayrak (1998), Adelman \& Gulliver (1990), Adelman \& Philip (1996). Adelman et al. (1997, 1999, 2000, 2001, 2004), Caliskan \& Adelman (1997), Kocer et al. (2003), and Pintado \& Adelman (2003). My conceptual model is that stars rotating sufficiently fast have normal abundances over all of their photospheres. At some point when the local rotation rate decreases below a critical value, polar regions begin to show peculiar abundances. As rotation further decreases the portion with normal abundances decreases until the abundances over the entire surface are peculiar, but not necessarily uniform.

The Si/Sr is a measure of the change of the anomalies with respect to atomic number $(Z)$. The solar value of $\log \mathrm{Si} / \log \mathrm{Sr}$ is 4.58 . A sign of the Am phenomena is the increase in the anomalies with $Z$ as illustrated in Figure 1 from Adelman et al. (2004) for the hot Am stars $\theta$ Leo and $o$ Peg. Lemke (1989) estimates errors in $T_{\text {eff }}$ of $\pm 150 \mathrm{~K}$ and in $\log g$ of \pm 0.2 dex. This suggests abundance errors of order 0.25 dex. The mean metallicities are not completely uniform as although they usually are based on most of the common elements there are wide differences in the detection and measurement of values for the less common elements and the spectral coverage differs from star to star. Although most of the HgMn stars are classified as B9 stars or close to this class, I include only $\nu$ Cnc, the coolest known HgMn star as an example since at abundance analysis spectral resolution this class is separable from the normal stars.

The stars are in order of increasing metallicity which correlates with $\log \mathrm{Fe} / \mathrm{H}$ and $\log \mathrm{Si} / \log \mathrm{Sr}$, expectations of the conceptual model. 29 Cyg is a $\lambda$ Boo star. Next are metal-poor stars, Vega, $\gamma$ Lyr, and $\sigma$ Boo. I break the distribution here and again at $\beta$ UMa, a known Am star. Stars with higher metallicities are Am stars except for 7 Sex, which behaves dynamically as a Population II star, and $\nu$ Cnc. The normal band is slightly asymmetric with respect to solar values. A few stars show non-solar Si/Sr ratios. Thus 29 Vul, 21 Lyn, and HR 6559 are incipient Am stars. The $\epsilon$ Aqr value may not be quite as well known due to its $v \sin i$ value. The errors in the analyses are sufficiently small for us to know that $\alpha$ Dra, at the low metallicity boundary is slightly metal-poor relative to the Sun and 5 Aqr at the high metallicity boundary is marginally metal-rich relative to the Sun. Table 4 samples stars with $v \sin i$ values between 6 and $110 \mathrm{~km} \mathrm{~s}^{-1}$. Within the normal star band the mean microturbulence is $1.6 \pm 1.3 \mathrm{~km} \mathrm{~s}^{-1}$ with 6 out 17 stars having values close to 0.0 .

The iron abundances of most of the stars agree with the previous classification. But $\eta$ Lep now is metal-poor while $15 \mathrm{Vul}$ has a mixture of underabundant and overabundant elements. This is also true of the 3 cited incipient Am stars. Thus among the sharp-lined A stars most do not have "solar" abundances.

\section{Acknowledgements}

I thank my fellow astronomers and collaborators who have guided me on the right path for the study of $\mathrm{A}$ and related type stars through discussions and by example. My adventures in A stars have been supported by grants from NSF, NASA, and The Citadel Foundation. I appreciate the observing time in the last 15 years particularly at the Dominion Astrophysical Observatory and with the Four College Automated Photoelectric Telescope, Fairborn Observatory, and at CASELO Observatory. 


\section{References}

Abt H. A. 1965, ApJS, 11, 37.

Abt H. A. 2000, ApJ, 544, 933.

Abt H. A. 2003, ApJ, 582, 420.

Abt H. A. 2004, ApJ, 603, L109.

Abt H. A. 1969, ApJ, 158, 1091.

Abt H. A., Morrell N. I. 1995, ApJS, 99, 135.

Abt H. A., Moyd K. I. 1973, ApJ, 182, 809.

Adelman S. J. 1986, A\&AS, 64, 173.

Adelman S. J. 1987, A\&AS, 67, 353.

Adelman S. J. 1989, MNRAS, 239, 487.

Adelman S. J. 1991, MNRAS, 252, 1161.

Adelman S. J. 1994, MNRAS, 271, 355.

Adelman S. J. 1996, MNRAS, 280, 130.

Adelman S. J. 1999, MNRAS, 310, 146.

Adelman S. J. 2001, IBVS, 5050.

Adelman S. J., Albayrak B. 1998, MNRAS, 300, 359.

Adelman S. J., Gulliver A. F. 1990, ApJ, 348, 712.

Adelman S. J., Philip A. G. D. 1996, MNRAS, 252, 1181.

Adelman S. J., Gulliver A. F., Heaton R. J. 2004, $A \& A$, in press.

Adelman S. J., Adelman A. S, Pintado O. I. 2003, A\&\&A, 397, 267.

Adelman S. J., Caliskan H., Kocer D., Bocal, C. 1997, MNRAS, 288, 470.

Adelman S. J., Caliskan H., Kocer D., Cay I. H., Tektunali H. G. 2000, MNRAS, 316, 514.

Adelman S. J., Caliskan H., Cay T., et al. 1999, MNRAS, 305, 601.

Adelman S. J., Caliskan H., Kocer D. et al. 2001, A\& A, 371, 1078.

Adelman S. J., Gulliver A. F., Smalley B., et al. 2004, These Proceedings, JP2

Andersen J. 1991, A\&AR, 3, 91.

Bessell M. S., Castelli F., Plex B. 1998, A\&AA,, 333, 231.

Blackwell, D. E., Lynas-Gray, A. E. 1994, A\& $A$, , 282, 899.

Bohlin R. C., Gilliland R. L. 2004, AJ, 127, 3508.

Breger, M. 2005, These Proceedings, 335.

Caliskan H., Adelman, S. J. 1997, MNRAS, 288, 501.

Code A. D., Davis J., Bless R. C, Hanbury Brown R. 1976, ApJ, 203, 417.

Conti P. S. 1970, PASP, 82, 781.

Gerbaldi M., Faraggiana R., Lai O. 2003, A\&A, 412, 447.

Girardi L., Bressan A., Bertelli, G., Chiosi C. 2000, A\&GAS, 141, 371.

Gray D. F. 1992, The Observation and Analysis of Stellar Photospheres, Cambridge University Press, Cambridge.

Gray R. O. 1988, AJ,, 95, 220.

Gulliver A. F., Hill G., Adelman S. J. 1994, ApJ, 429, L81.

Harmanec P. 1988, Bull. Astron. Inst. Czechosl., 39, 329.

Hauck B., Slettebak A. 1983, A\&AA, 127, 231.

Hayes D. S., Latham D. W. 1975, ApJ, 197, 593.

Hill G., Fisher W. A. 1986, Pub. Dom. Astrophys. Obs.16, No. 13.

Hill G., Gulliver A. F., Adelman S. J. 2005, These Proceedings, 35

Hoffleit D., Warren W. H., Jr. 1991, The Bright Star Catalogue, 5th Rev. Ed., ADC Selected Astronomical Catalogs, Vol. 1, NASA Goddard Space Flight Center.

Kocer D., Adelman S. J., Caliskan H., Gulliver A. F., Tektunali H. G. 2003, A\&\&A, 406, 975.

Kurucz R. L., Avrett E. H. 1981, SAO Special Report No. 191.

Landstreet J. D. 1998, A\&A, 338, 1041.

Lemke M. 1989, $A \& \exists A, 225,125$.

Lutz T. F., Kelker D. H. 1973, PASP, 85, 573.

Monnier J. D. 2003, Rep. Prog. Physics, 66, 789. 
Morgan W. W., Keenan P. C., Kellman E. 1943, Atlas of Stellar Spectra, University of Chicago Press, Chicago.

Pagano I., Linsky J. L., Valenti J., Duncan D. K. 2004, A\&A, 415, 331.

Pintado O. I., Adelman S. J. 2003, A\&A, 406, 987.

Popper D. M. 1980, ARA\&A, 18, 115.

Preston G. W. 1974, ARA\&A, 12, 257.

Rachford B. L. 1998, ApJ, 505, 255.

Reiners A., Royer F. 2004, A\&A, 415, 325.

Roman N. G., Morgan W. W., Eggen O. J. 1948, ApJ, 110, 205.

Schmitt J. H. M. M. 1997, A\&BA, 318, 215.

Schaller G., Schaerer D., Meynet G., Maeder A. 1992, A\&SAS, 96, 269.

Shorlin S.L.S., Wade G. A., Donat J.-F., Landstreet J. D., et al. 2002, A\&GA, 392, 637.

Simon T., Landsman W. B. 1991, ApJ, 380, 200.

Simon T., Landsman W. B. 1997, ApJ, 483, 435.

Smalley B., Dworetsky M. M. 1995, A\&A, 293, 446.

Smalley B., Gardiner R. B., Kupka, F., Bessell M. S. 2002, A\&A, 395, 601.

Tüg H., White N. M., Lockwood G. W. 1977, A\&A, 61, 679.

Turner-Bey D. E., Kashyap V., Evans N. et al. 2003, AAS Meeting 203, \#85.03.

van Belle G. T., Ciardi D. R., Thompson R. R. et al. 2001, ApJ, 559, 1155.

Waelkens C., Aerts C., Kestens E. et al. 1998, A\&\&A, 330, 215.

Wolff S. C. 1983, The A-Stars: Problems and Perspectives, NASA SP-463, Washington, DC.

\section{Discussion}

L. BALOnA: You mentioned that one of the main characteristics separating normal and peculiar stars are the $v \sin i$ values. Yet a brief scan of $v \sin i$ in your table does not suggest any difference in $v \sin i$ between them.

S. Adelman: My stars were chosen to be relatively sharp-lined to make the elemental abundance analyses easily done. I agree that is very important to obtain abundances of stars with rotation. In this regard, I note the pioneering work of Grant Hill.

T. RyABChikova: If we find a low $v \sin i$ normal abundance star, does it mean that star should necessarily be pole-on fast rotator like Vega?

S. Adelman: Most likely. It would be useful to examine the model of Vega to see for what values of $i$ there are flat-bottomed weak lines.

J. LANDSTREeT: You have chracterized convection as being first clearly apparent around A5. However, as you decrease effective temperature along the main sequence, non-zero values of microturbulence parameter are first seen about A0. This seems to me to be pretty clear indication of convection at least in the deep atmosphere.

S. Adelman: I agree that the non-zero microturbulence found in the early A stars is an indicator of incipient convection.

C. Corbally: What kinds of differences are found for published $v \sin i$ values derived by the Fourier technique and your line profile determinations.

S. Adelman: For most cases the agreement is good to excellent. But it is the outlyers which worry Austin Gulliver and me. 